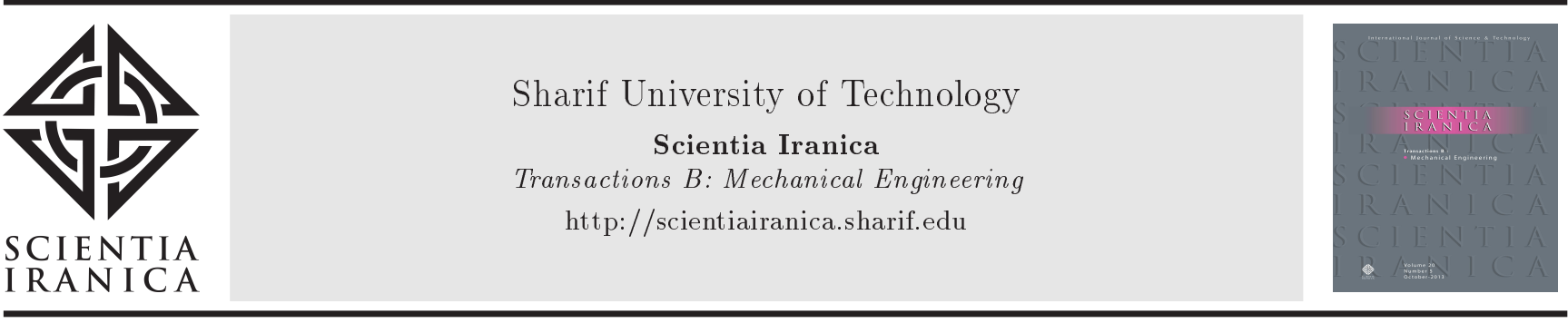

\title{
Damage detection in a double-beam system using proper orthogonal decomposition and teaching-learning based algorithm
}

\author{
A. Mirzabeigy and R. Madoliat* \\ School of Mechanical Engineering, Iran University of Science and Technology, Narmak, Tehran, 16846, Iran.
}

Received 26 February 2018; received in revised form 8 August 2018; accepted 3 December 2018

\author{
KEYWORDS \\ Double-beam system; \\ Proper orthogonal \\ decomposition; \\ Crack; \\ Damage; \\ Teaching-learning \\ based optimization.
}

\begin{abstract}
This study applies the inverse approach to damage detection in a doublebeam system. A double-beam system made of two parallel beams is connected through an elastic layer. Degradation of the stiffness of beams element, crack occurrence, and partial destruction of the inner layer are considered as different types of damage. The time domain acceleration response of the system is measured, and proper orthogonal decomposition is applied to the collected data in order to derive Proper Orthogonal Values (POVs) and Proper Orthogonal Modes (POMs) of the system. Effect of single damage in different locations on the POV has been analyzed, and an objective function is defined using the dominant POV and POM of each beam separately. In order to increase the robustness of the method against noise, the objective function is enriched by adding statistical property of time domain response. The teaching-learning based optimization algorithm is employed to solve the optimization problem. The efficiency of the proposed method for detecting single and multiple damages in the system is demonstrated with and without noise. Simulation results verify the good accuracy of the proposed method for detecting single and multiple damages of different types in the system.
\end{abstract}

(C) 2020 Sharif University of Technology. All rights reserved.

\section{Introduction}

Health monitoring of structures and on-time damage detection are two common issues in mechanical, civil, and aerospace engineering and are used for preventative measures to avoid human casualties and financial detriment. Several methods have been developed to detect damage to the structures. One of the best of these methods is vibration-based damage detection, which is widely used because of its low cost, the

\footnotetext{
*. Corresponding author.

E-mail addresses: mirzabeigy@mecheng.iust.ac.ir (A. Mirzabeigy); r_mdlt@yahoo.com; madoliat@iust.ac.ir (R. Madoliat)
}

doi: $10.24200 /$ sci. 2019.50520 .1738 ability to monitor the whole structure by measuring a few limited points, and non-requirement for the presence of a skillful operator. The main idea used in vibration-based damage detection is the change in the modal parameters such as natural frequencies, mode shapes, and damping ratio of the structure after damage occurrence. Further, the dynamic response or forced vibration response of the structure is affected by damage and becomes different. In a general category, vibration-based methods for damage detection are divided into two types: model-based and responsebased methods. In a model-based method, a numerical or analytical model of the structure is available in a response-based method, and only the structure response to the excitation or modal parameters is available irrespective of its model. A comprehensive review of different techniques for structural damage 
detection using vibration analysis was presented by Fan and Qiao [1].

One of the popular methods for damage detection that uses the model of the structure is inverse approach. In the inverse approach, the model of the structure has been updated using modal parameters or dynamic response; any probable damage can be identified through model updating. For this purpose, an objective function and an optimization algorithm are required. The objective function is defined as the discrepancy between modal parameters or dynamic response of the model of the structure and the monitored structure. Whenever the objective function value becomes zero or very small, the damage parameters are detected. Further, a fast and stable optimization algorithm is required to find a unique solution for the objective function. Several studies have designated damage detection as an inverse problem. Ruotolo and Surace [2] defined an objective function based on natural frequencies, modal curvature, and normalized mode shapes for crack detection in the beam-like structure, and the genetic algorithm was used to solve the optimization problem. Meurane and Heylen [3] applied an objective function, which is the summation of the five fundamental modal parameters based on objective functions to detect damage in a three-dimensional, statically indeterminate space truss. Raich and Liszkai [4] proposed an objective function through the frequency response function to detect damage in structures. Other research pieces in which damage detection has been formulated as an inverse problem could be found in [5-12].

Sandwich beams have many applications in aerospace and naval industries, as well as bridges and buildings construction. The sandwich beams are made of three layers: two faces characterized by thinness and high strength and inner layer characterized by thickness, light weight, and low stiffness. Desired structural properties such as high stiffness and low weight are achieved by combining the strong face with a thick and low-density core. A double-beam system consists of two parallel beams with a Winklertype elastic inner layer, considered as an approximate model for the sandwich beam. The main difference between a soft-core sandwich beam and a doublebeam system lies in the former's disregard for the role of shear deformation of the core in the simplified model. The dynamic characteristics of the doublebeam system have been investigated in several papers. Oniszczuk [13] investigated the free vibrations of the double-beams system, which are continuously joined by a Winkler elastic layer with simply support boundary conditions, and presented an analytical formulation for natural frequencies. Palmeri and Adhikari [14] proposed a Galerkin-type state space for transverse vibrations of a double beam with a viscoelastic inner layer. Stojanovic et al. [15] applied Timoshenko and high-order shear deformation theory to deal with the problem of free transverse vibration and buckling of an elastically connected simply supported set of beams under the compressive axial load. Other studies in this field could be found in [16-18]. In these studies, the beams and core remained intact, and effects of damage were neglected. Mirzabeigy and Madoliat [19] investigated the free vibration of the partially connected double-beam system. In fact, their study deals with the effect of damage in the inner elastic layer on natural frequencies and mode shapes. Results in [19] showed that for similar boundary conditions of upper and lower beams, damage to the core did not affect odd mode frequencies. Nguyen [20] addressed the effect of crack on the double-beam system by applying Finite Elements (FE) method. For crack detection, Nguyen [20] utilized the wavelet transform of the relationship between the natural frequency and the location of a concentrated mass, which was located on the main beam. There was no other report about damage detection in a double-beam system, except for damage reports in [20].

In this study, Proper Orthogonal Decomposition (POD) is applied to damage detection in a doublebeam system. POD only uses data from the timedomain response and does not need any frequency domain analysis. In addition, this method involves data fusion between data from several sensors and reduces the volume of data by extracting useful information. An attempt is made here to define an appropriate objective function for damage detection by using POD and increase the method's robustness against measurement noise. The paper is organized as follows: the finite element model of the doublebeam system in the presence of different damages is formulated in Section 2. Section 3 is devoted to the procedure of Teaching-Learning Based Optimization (TLBO) algorithm. Section 4 introduces the POD; the main idea, history, mathematical formulation, and application of damage detection are illustrated. Section 5 is related to damage detection; at first, an objective function is defined by considering effects of different kinds of damage and robustness against noise, Then, damage detection results with and without noise are presented, and the effects of different parameters on the convergence rate are investigated.

\section{The system modeling}

Consider two parallel beams with the Winkler-type elastic inner layer in clamp-free boundary conditions, as depicted in Figure 1. A time-varying point force is exerted on the free end of an upper beam. It is assumed that beams are thin and can be model by the Euler-Bernoulli beam theory. The beams have the same rectangular cross-section, material properties, 


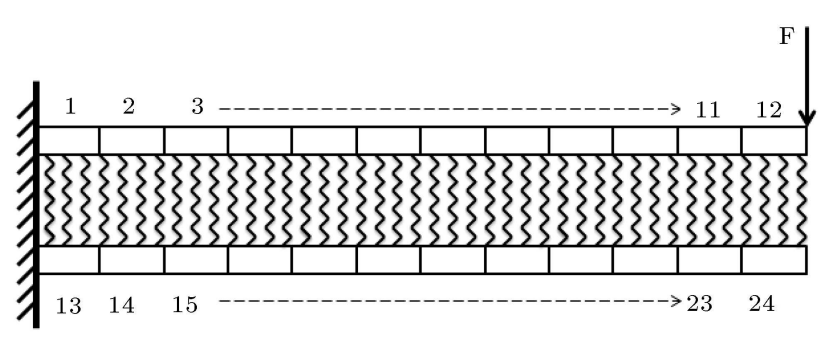

Figure 1. Two parallel beams connected through an elastic layer.

and length. The beam's modulus of elasticity, material density, height, width, and length are $E, \rho, h, w$, and $L$, respectively. By applying the geometry of crosssection, the area and moment of inertia are calculated and considered as $A$ and $I$. The coefficient of Winkler elastic layer is $k$. Two independent coordinates considered for beam deflection are denoted by $y_{1}$ and $y_{2}$, while the horizontal coordinate for both beams is the same, as denoted by $x$. The equations of motion for this system are as follows:

$$
\begin{aligned}
& E I \frac{\partial^{4} y_{1}(x, t)}{\partial x^{4}}+\rho A \frac{\partial^{2} y_{1}(x, t)}{\partial t^{2}}+k\left[y_{1}(x, t)-y_{2}(x, t)\right] \\
& =F(t) \delta(x-L),
\end{aligned}
$$

$$
\begin{aligned}
& E I \frac{\partial^{4} y_{2}(x, t)}{\partial x^{4}}+\rho A \frac{\partial^{2} y_{2}(x, t)}{\partial t^{2}}+k\left[y_{2}(x, t)-y_{1}(x, t)\right] \\
& =0
\end{aligned}
$$

Solving Eqs. (1) and (2) in the analytic form is not easy and will be onerous in case of any damage to the structure. Therefore, a numerical scheme like FE is adopted for this problem. A two-node thin beam element with two degrees of freedom in each node is applied to system discretization. The mass and stiffness matrix for this element could be found in different books like [21]. The two beams are related to each other via an elastic inner layer, as observed in the schematic sketch of the system and equations of motion. This elastic layer does not add any new degree of freedom to the system and only has a stiffness matrix in the FE formulation, because its mass is neglected. The stiffness matrix of the inner layer is the stiffness derived for the Winkler elastic layer. In the present system, displacement vectors or degrees of freedom vector of the upper and lower beams are $\left\{d_{1}\right\}$ and $\left\{d_{2}\right\}$, respectively. The general displacement vector is presented as follows:

$$
\{D\}=\left\{\begin{array}{l}
d_{1} \\
d_{2}
\end{array}\right\} .
$$

For each beam, the global stiffness matrix due to bending is $K_{B}$, global mass matrix of beam is $M_{B}$,

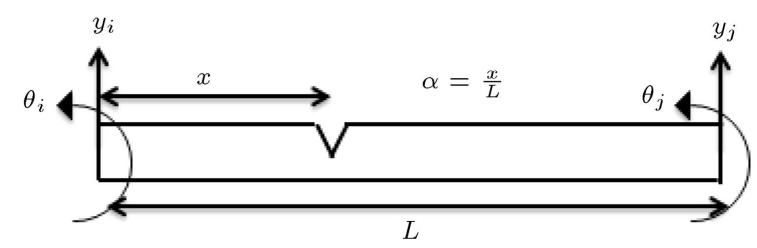

Figure 2. The beam element with a transverse crack.

and global stiffness matrix due to the Winkler layer is $K_{W}$. Finally, a general equation of system is as follows:

$$
\begin{aligned}
& {[K]\{\ddot{D}\}+[M]\{D\}=\{F\},} \\
& {[K]=\left[\begin{array}{cc}
K_{B}+K_{W} & -K_{W} \\
-K_{W} & K_{B}+K_{W}
\end{array}\right],} \\
& {[M]=\left[\begin{array}{cc}
M_{B} & 0 \\
0 & M_{B}
\end{array}\right] .}
\end{aligned}
$$

The time history response of the discretized system in Eq. (4) can be calculated using implicit algorithm called Newmark's method [22].

Modal parameters of the system such as natural frequencies are obtained by the harmonic motion assumption with a circular frequency of $\omega$ and neglecting external force, leading to the following:

$$
\left([K]-\omega^{2}[M]\right)\{D\}=0 .
$$

Eq. (5) is an eigenvalue problem, in which, for the nontrivial solution, it is necessary that the determinant of the coefficient matrix be set equal to zero. The obtained eigenvalues correspond to the natural frequencies of vibration.

Three different types of damage are considered in this study: One is the modulus of elasticity degradation of a specific element. If damage intensity is denoted by $\beta$, then the modulus of elasticity is considered as $E^{d}=(1-\beta) E$ for constructing stiffness matrix for this element, where superscript $d$ represents the damage state. Another type of damage is crack occurrence. Effect of the crack on the stiffness matrix of a thin beam element was formulated by Mehrjoo et al. [23]. Figure 2 shows a beam element with transverse crack. Dimensionless crack location is denoted by $\alpha$, and dimensionless crack severity represents the crack depth to beam height ratio, as denoted by $\eta$. The equivalent spring stiffness for this crack based on the fracture mechanics principles is given as follows [24]:

$$
\begin{aligned}
K_{C}= & \frac{E w h^{2}}{72 \pi f(\eta)} \\
f(\eta)= & 0.6384 \eta^{2}-1.035 \eta^{3}+3.7201 \eta^{4}-5.1773 \eta^{5} \\
& +7.553 \eta^{6}-7.332 \eta^{7}+2.491 \eta^{8}
\end{aligned}
$$




$$
\begin{aligned}
& {\left[k_{C}\right]=\frac{-1}{B L^{2}}\left[\begin{array}{cccc}
(2 A+C A+1) & (A+1) L & -(2 A+C A+1) & (A+A C) L \\
& L^{2} & -(A+1) L & A L^{2} \\
& & (2 A+C A+1) & -(A+A C) L \\
& & & C A L^{2}
\end{array}\right]} \\
& A=\frac{K_{C} L+6 E I \alpha(1-\alpha)}{2 K_{C} L+6 E I \alpha^{2}}, \quad B=(A-1) \frac{L}{2 E I}+(A+1) \frac{\alpha}{K_{C}}-\frac{1}{K_{C}}, \quad C=\frac{2 K_{C} L+6 E I(1-\alpha)^{2}}{K_{C} L+6 E I \alpha(1-\alpha)} .
\end{aligned}
$$

Box I

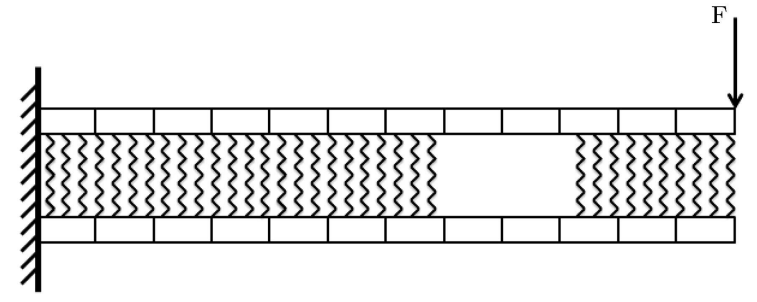

Figure 3. Damage in the core as partial destruction of the elastic inner layer.

The stiffness matrix for the cracked element is presented in Eq. (7) [20] as shown in Box I. The last possible damage type is inner layer damage. Damage is assumed to be complete removal of elastic inner layer in a specific range, as shown in Figure 3. Stiffness matrix assembly is important in this type of damage, because the stiffness matrix due to the Winkler elastic layer is zero for the elements in the damage region.

\section{Teaching-Learning Based Optimization (TBLO)}

TLBO is a population-based, metaheuristic algorithm inspired by the education procedure in a classroom. TLBO was proposed by Rao et al. [25] and applied to different areas of science and engineering [26-30]. The logic behind TLBO is that learners (students) seek better grades through education; therefore, teacher has a superior role. A good teacher could educate learners better and help raise their grades. The teacher's teaching quality could be evaluated through the mean value of class scores, and the best teacher is he/she whose knowledge equals the mean value of his class; in other words, the teacher and learners have the same amount of knowledge. In addition to the education made possible by the teacher, learners could educate themselves through collaboration and reciprocate knowledge. TLBO simulates teacher-based education and collaborative learning among students for finding global optimum. TLBO is parameterfree and does not need any parameter tuning; it requires only common controlling parameters such as population size and number of generations for its working. In this algorithm, different decision variables are analogous to different subjects taught to students, and the students' overall result is analogous to the values of the objective function. The TLBO procedure is divided into two phases: teacher phase and learner phase.

\subsection{Teacher phase}

The first step of knowledge sharing in the TBLO is the teacher phase, which attempts to simulate the teacher's influence on the student. In this phase, the learners or students are motivated by the teacher and attempt to promote their knowledge, which consequently yields an increase in the mean result of the class concerning the subject matter taught by the teacher. Consider $N$ number of learners in a class and $J$ number of subject matters being taught to them. They represent the population size of $N$ with $J$ design variables. At any iteration, the mean result of the class in a specific subject $j(1,2,3, \ldots, J)$ is given by:

$$
M_{j}=\frac{1}{N} \sum_{k=1}^{N} X_{k}(j),
$$

where $X_{k}(j)$ is the grade of learner $k$ in subject $j$.

The learner with the best overall grade of all the subjects (or best fitness value) is designated as the teacher, and other learners progress alongside the teacher to enhance their own overall grades through the following equation:

$$
X_{k}^{\text {new }}(j)=X_{k}^{\text {old }}(j)+r\left(X_{\text {Teacher }}(j)-T_{F} M_{j}\right),
$$

where $X_{k}^{\text {new }}(j)$ and $X_{k}^{o l d}(j)$ are the new and old grades of learner $k$ in subject $j ; r$ is the random number in the range $[0,1] ; X_{\text {Teacher }}(j)$ is the teacher grade in subject $j$; and $T_{F}$ is teaching factor, which could be either 1 or 2 with an equal level of probability. $X_{k}^{\text {new }}$ will be accepted instead of $X_{k}^{\text {old }}$ if it gives a better fitness function. At the end of each teaching cycle, the current best student will become the teacher for the next iteration.

\subsection{Learner phase}

Having gained the necessary knowledge under teacher conduction, learners could be entitled to a greater 
degree through mutual discussion and interactive learning. In this phase, for each student $p$ from the class, another student $q$ is randomly select in such a way that $p \neq q$. Following the fitness evaluation of both students, if student $p$ is better than student $q$, then:

$$
\begin{aligned}
& X_{p}^{\text {new }}(j)=X_{p}^{\text {old }}(j)+r\left(X_{p}^{\text {old }}(j)-X_{q}(j)\right), \\
& j=1,2, \ldots, J
\end{aligned}
$$

otherwise:

$$
\begin{aligned}
& X_{p}^{\text {new }}(j)=X_{p}^{\text {old }}(j)+r\left(X_{q}(j)-X_{p}^{\text {old }}(j)\right), \\
& j=1,2, \ldots, J
\end{aligned}
$$

where $r$ is the random number in the range $[0,1] . X_{p}^{n e w}$ will be accepted rather than $X_{p}^{\text {old }}$ if it gives a better fitness function.

In analogy with an optimization problem, the grade of the $k$ th student in subject $j$ or $X_{k}(j)$ is the value of design variable $j$ for the $k$ th possible solution. The overall grade of each student corresponds to the fitness evaluation of each possible solution to the optimization problem. Fitness evaluation is dependent on the objective function definition in the optimization problem.

\section{The Proper Orthogonal Decomposition (POD)}

The POD is a multi-variate statistical procedure applied to derive a compact representation of the data. This method may serve two purposes, namely order reduction by projecting highly dimensional space to a space of lower dimension and feature extraction by revealing a relevant, but unexpected, structure hidden in the data $[31,32]$.

The practical application of the POD consists of the acquisition of data of a vibrating system over a definite period of time $T$. Measured data (acceleration, velocity, or displacement) of a vibrating structure are varying in time and space; therefore, data are represented in the matrix form. In each location, the recorded values of data are uniformly distributed in time. It is possible to construct an $N \times M$ matrix, where each row represents a snapshot of the system at a certain instant, while each column shows the time history of a single measurement point. The observation matrix $V$ contains spatial information of the system in time as follows:

$$
V=\left[\begin{array}{cccc}
v_{1}\left(t_{1}\right) & v_{2}\left(t_{1}\right) & \ldots & v_{M}\left(t_{1}\right) \\
v_{2}\left(t_{2}\right) & v_{2}\left(t_{2}\right) & \ldots & v_{M}\left(t_{2}\right) \\
\cdot & \cdot & \ldots & \cdot \\
\cdot & \cdot & \ldots & \cdot \\
\cdot & \cdot & \ldots & \cdot \\
v_{1}\left(t_{N}\right) & v_{2}\left(t_{N}\right) & \ldots & v_{M}\left(t_{N}\right)
\end{array}\right] .
$$

A new matrix is constructed in which all elements of the $i$ th column are the same and equal to the average value of the $i$ th column of $V$. This new matrix is called $W$. In order to perform POD, A matrix $U$ is generated by subtracting $W$ from $V$ as follows:

$$
U=V-W
$$

Finally, the correlation matrix is formed as follows:

$$
R=\frac{1}{N} U^{T} U
$$

where $R$ is a real, square and symmetric matrix of dimension $M$. Like any real, square matrix of dimension $M$, there are $M$ eigenvalues and eigenvectors that exist for $R$. The eigenvalues of $R$ are called Proper Orthogonal Values (POVs), and the eigenvectors of $R$ are named Proper Orthogonal Modes (POMs). Feeny and Kappagantu [33] demonstrated that for a structure with uniform mass distribution in the free vibration state, linear natural modes closely correlate with POMs, and POVs represent the energy associated with each mode. The relationship between POMs and deformed configuration (superposition of linear natural modes) in forced vibration was shown by Kerschen and Golinval [34]. This relationship between linear natural modes and POMs was also recognized in the case of randomly excited structures by Feeny and Liang [35]. The natural modes of structures are very sensitive to damage and provide spatial information about the structure state; further, POD method, known as a data reduction method, affords a sort of information that is closely related to natural modes; thus, POD outcome could be applied to damage detection for structures. In the case of a structure whose necessary data are acquired at $M$ location, the possible number of POMs that could be obtained is $M$. It is reasonable to state that if the number of $M$ increases, POMs provide a more accurate dynamic shape of a structure. One problem with respect to $\mathrm{POD}$ is the importance value of each POM and POV or, in other words, how much information is provided by each POM. The goal is to use the minimum number of POMs and POVs to achieve maximum data. One of the methods applied by several researchers is energy criterion. POV gives the value of energy associated with the corresponding proper orthogonal so as to determine the role of each mode in the whole data. The required number of POMs for an accurate description of a structure dynamic is determined by the smallest value of number $j$, which satisfied the following energy criterion:

$$
\frac{\sum_{k=1}^{j} \lambda_{k}}{\sum_{k=1}^{M} \lambda_{k}}>0.99,
$$


where $\lambda$ is the POV and sorted in descending order so that $\lambda_{1}>\lambda_{2}>\ldots>\lambda_{M}$. In many cases, especially when harmonic excitation with a frequency close to one of resonance frequencies of the structure is exerted, energy criterion will be satisfied by the first POV or $j=1$.

Galvanetto and Violaris [36] applied POM to damage detection. By applying energy criterion in Eq. (15), the dominant POM of the structure before and after damage occurrence is determined, and the following damage indicator is used:

$$
\begin{aligned}
& \triangle P O M(i)=P O M_{u}(i)-P O M_{d}(i), \\
& i=1,2, \ldots, M
\end{aligned}
$$

where $\mathrm{POM}_{u}(i)$ is the dominant proper orthogonal mode before a damage or undamaged case at the $i$ th measurement point, and $P O M_{d}(i)$ is the dominant proper orthogonal mode after damage at the same point. Damage location is revealed by an abrupt change in the graph of $\triangle P O M$. This method is applied to damage detection in uniform beams. Galvanetto et al. [37] verified the results in [36] via the experimental test.

Using curvature of $\triangle P O M$, Thiene et al. [38] increased the accuracy of the method, presented in [36]. The curvature is calculated for the dominant POM using the central difference method as follows:

\section{Curvature $=$}

$$
\frac{P O M(i+1)+P O M(i-1)-2 P O M(i)}{H^{2}},
$$

where $H$ is the uniform grid spacing. Possible damage location is detected using the difference in the curvature of POM before and after damage occurrence. This method is applied numerically to the composite beam and experimentally to an aluminum plate. Rao et al. [39] defined an optimization problem using POMs and POVs.

\section{Damage detection}

\subsection{Methodology}

A double-beam system with the Winkler-type elastic inner layer has been considered. Boundary conditions of the structure are clamp-free, and the harmonic load is applied to the free end of the upper beam. Both beams are similar, and the physical and geometrical

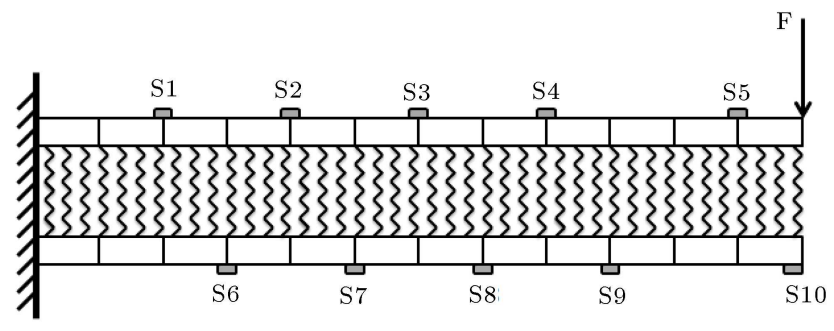

Figure 4. Configuration of the 10 measurement points in the double-beam system.

properties of the system are as follows:

$$
\begin{aligned}
& E=200 \mathrm{GPa}, \quad \rho=7600 \mathrm{~kg} / \mathrm{m}^{3}, \quad h=60 \mathrm{~mm}, \\
& w=90 \mathrm{~mm}, \quad k=10^{5} \mathrm{~N} / \mathrm{m}^{2}, \quad L=1.5 \mathrm{~m},
\end{aligned}
$$

where $E, \rho, h, w, k$, and $L$ are the modulus of elasticity, density, beam's height, beam's width, inner layer stiffness, and length, respectively. Each beam is divided into 12 elements, and the acceleration response of the structure measured at 10 locations $(S 1, S 2, \ldots, S 10)$ is depicted in Figure 4. The first four natural frequencies of the system are calculated using Finite Element Method (FEM) and presented in Table 1. It is observed that the first and second frequencies are close to the third and fourth frequencies, because the stiffness of the elastic inner layer is lower than that of beams.

Now, the effects of damage on the proper orthogonal value of a system are investigated, as shown in Figure 4. Harmonic load with a frequency excitation rate of 10 Hertz is considered as follows:

$$
F=2000 \sin (20 \pi t) .
$$

The response of the system is recorded over a time duration of 3 seconds after the excitation exerted on the system. There are 10 sensors installed on the system; therefore, 10 POVs could be achieved. As mentioned earlier, dominant POV and POM provide more information about the system; consequently, only one POV with a maximum value is used. Damage is assessed to be $10 \%$ degradation of the modulus of elasticity of one element. Effects of detected damage to the upper beam on the dominant POV are depicted in Figure 5. In this graph, horizontal axes are related to damage location, where damage elements 1 and 12 are elements at the clamped end of the upper beam and the free end of upper beam, respectively. Other elements are located between these two elements and numbered from the clamped end to the free end, respectively. It is clear that the value of POV decreases when damage

Table 1. The first four natural frequencies of the system in Hertz.

\begin{tabular}{cccc}
\hline First frequency & Second frequency & Third frequency & Fourth frequency \\
\hline 22.098 & 24.734 & 138.48 & 138.93 \\
\hline
\end{tabular}




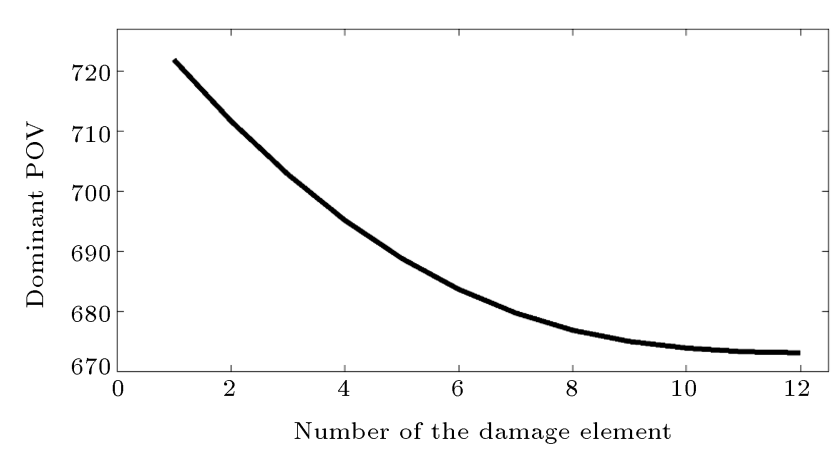

Figure 5. The effects of damage at the upper beam on the dominant Proper Orthogonal Value (POV).

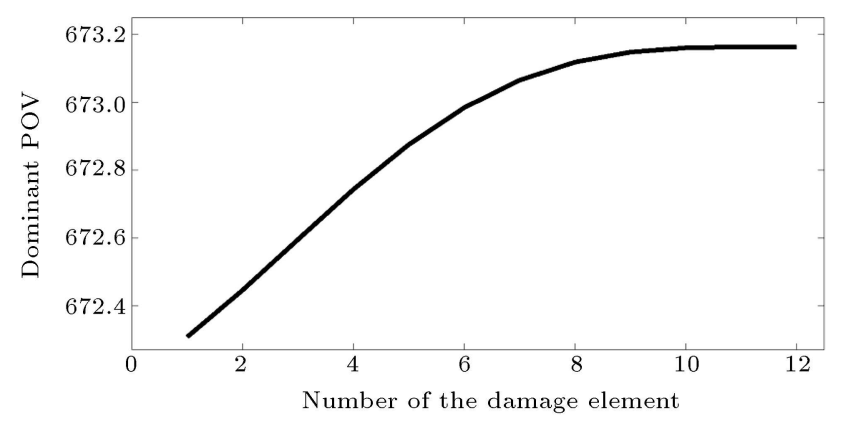

Figure 6. Effects of damage at the lower beam on the dominant Proper Orthogonal Value (POV).

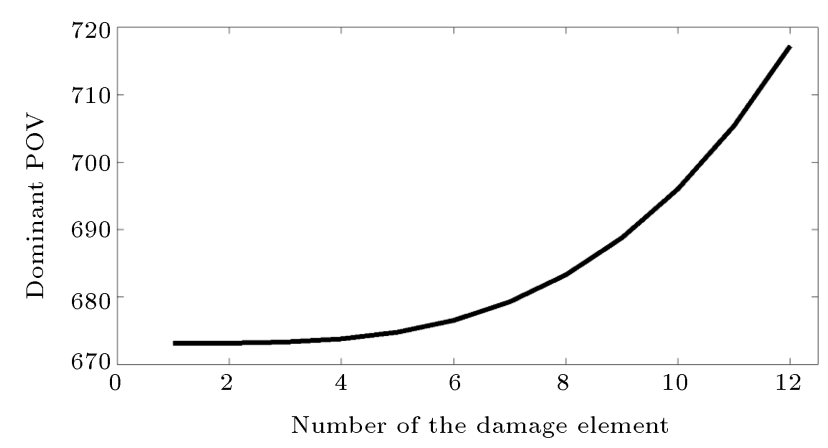

Figure 7. Effects of damage at the core on the dominant Proper Orthogonal Value (POV).

approaches the free end and, also, the variation of POV near the free end is quite minor. Effects of damage to the lower beam on the dominant POV are shown in Figure 6. Element numbering in the case of the lower beam is similar to that in the upper beam where damage element 1 is an element at the clamped end. Variations of POV with damage to the lower beam are quite small, and the difference between maximum and minimum variations is less than $0.2 \%$, while damage to the upper beam causes about $7 \%$ variation in POV. Effects of damage location in the inner elastic layer on the dominant POV are shown in Figure 7. Damage is considered to be the complete removal of the inner layer between the two elements. In this case, the damage element $k$ is related to removing the inner layer between element $k$ of the upper beam and element $k$ of the lower beam. As observed earlier, the POV value increases when the inner layer damage approaches the free end. The value of POV when damage occurs at the free end of the upper beam is very close to the value of POV when damage occurs in the elastic layer near the clamped end. Moreover, the value of POV when damage occurs at the clamped end of the upper beam is very close to that of POV when damage occurs in the elastic layer near the free end. This issue may mislead and complicate the damage detection procedure, and the detected damage to the elastic layer is identified instead of the damage to the upper beam, and vice versa. To obviate this problem, POVs of each beam are calculated separately, and effects of damage on the dominant POV are examined. Each beam has five POVs individually. The dominant POVs of the Upper and Lower beams are called POVU and POVL, respectively. Effects of damage on the upper beam, lower beam, and elastic inner layer on POVU and POVL are depicted in Figures 8, 9, and 10, respectively. Different trends observed for the variation of POVU and POVL with different cases of damage are remedial for damage detection; for instance, the application of POVU and POVL makes it possible to differentiate between the damage to the upper beam near clamped end and the damage to elastic inner layer near free end. As seen, the calculation of the POV for each beam separately provides more information about the system rather than POV calculation for the whole system. By applying POVU and POVL, an objective function is defined for damage detection in the double-beam system as follows:

$$
O b j_{1}=\left|1-\frac{P O V U^{m}}{P O V U^{*}}\right|+\left|1-\frac{P O V L^{m}}{P O V L^{*}}\right|,
$$

where $P O V U^{m}$ and $P O V L^{m}$ are the dominant POVs of the upper and lower beams from FE model, respectively. $P O V U^{*}$ and $P O V L^{*}$ are the dominant POVs of upper and lower beams of the system, respectively, with unknown damage, acquired either experimentally or by the numerical model.

Another objective function could be found by using the dominant POM of the upper and lower beams as follows:

$$
\begin{aligned}
O b j_{2}= & \sum_{j=1}^{M U}\left|1-\frac{P O M U^{m}(j)}{P O M U^{*}(j)}\right| \\
& +\sum_{k=1}^{M L}\left|1-\frac{P O M L^{m}(k)}{P O M L^{*}(k)}\right|,
\end{aligned}
$$

where $P O M U^{m}$ and $P O M L^{m}$ are the dominant POMs of the upper and lower beams from FE model, respectively. $P O M U^{*}$ and $P O M L^{*}$ are the dominant 

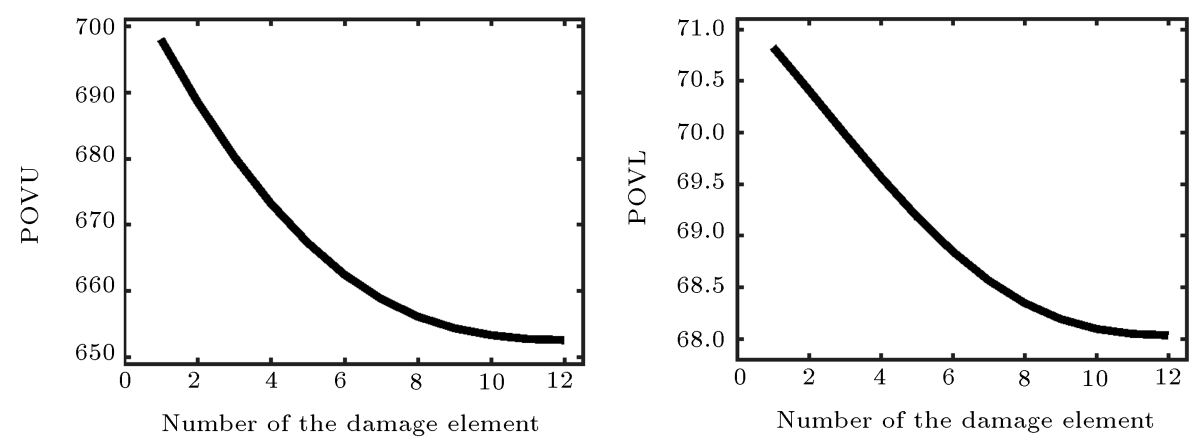

Figure 8. Effects of damage at the upper beam on the Proper Orthogonal Value of Upper and Lower beams (POVU and POVL).
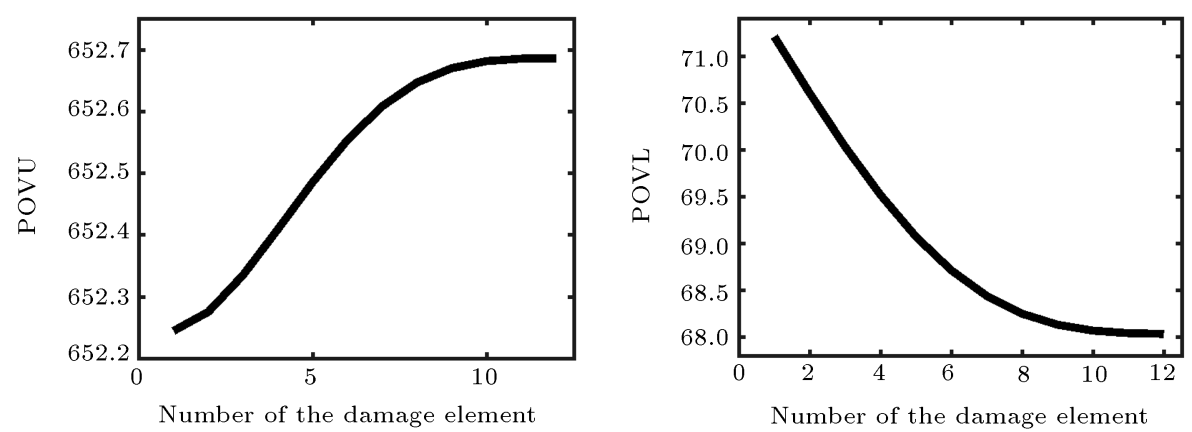

Figure 9. Effects of damage at the lower beam on the Proper Orthogonal Value of Upper and Lower beams (POVU and POVL)
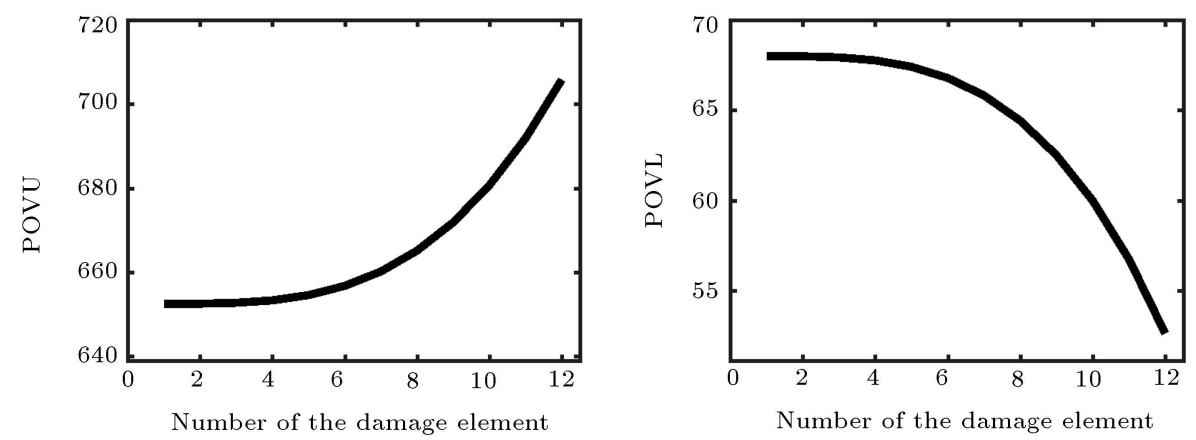

Figure 10. Effects of damage at the core on the Proper Orthogonal Value of Upper and Lower beams (POVU and POVL)

POMs of the upper and lower beams of the system with unknown damage, respectively. $M U$ and $M L$ are the number of measurement points at the upper and lower beams, respectively. It is clear that the goal of this objective function is to minimize the difference between dominant POMs of numerical data and available data from the system with unknown damage. Both of the proposed objective functions for damage detection are the same as the function of the structure model updating procedure. During the model updating process, parameters of damage such as location and severity will be identified.

In real applications, the noise effect is a menace that can reduce the precision of the damage detection strategy. In the real measurement, noise or error in data acquisition is inevitable. In order to account for the measurement noise, simulated data are contaminated by the addition of normally distributed random errors with the mean zero to ideal data as follows:

$$
v_{i}^{N o i s e}=v_{i}^{\text {Ideal }}+N L \times R n d m \times s t d\left(v_{i}^{I \text { deal }}\right),
$$

where $v_{i}^{\text {Noise }}$ and $v_{i}^{\text {Ideal }}$ are the noisy and ideal data at the $i$ th measurement point, respectively. $N L$ is the Noise Level, $R n d m$ is the random vector with zero mean, and all elements of this vector are the random values between $[-11]$, and $s t d\left(v_{i}^{\text {Ideal }}\right)$ is the standard deviation of the ideal data time history. The robustness of the damage detection strategy against noise is an important problem and, often, researchers 
claim that their strategy is robust enough against noise and that there is no need for any modifications. In the present work, the damage detection procedure becomes more robust against noise by using principal properties considered for noise. As mentioned, noise is a random vector with mean zero. It is quite simple to show that the addition of a zero mean vector to a vector with a mean value of $\alpha$ yields a new vector with a mean value of $\alpha$. In Eq. (21), the addition of the zero mean noise does not change the mean value, and both $v_{i}^{\text {Noise }}$ and $v_{i}^{\text {Ideal }}$ have the same mean value. Invariant behavior of the mean value in Eq. (21) makes it suitable to construct a new objective function as follows:

$$
O b j_{3}=\sum_{i=1}^{M}\left|1-\frac{\operatorname{mean}\left(v_{i}^{m}\right)}{\operatorname{mean}\left(v_{i}^{*}\right)}\right| .
$$

As illustrated, there is no difference in the mean value of recorded responses with or without noise; therefore, the mean value of either noisy or ideal data could be used in Eq. (22). mean $\left(v_{i}^{m}\right)$ and mean $\left(v_{i}^{*}\right)$ are the mean values of data recorded at the $i$ th measurement point obtained from the numerical model and available data from system with unknown damage, respectively.

Probabilistic damage will be evaluated by using an objective function, which is the sum of the mentioned objective functions as follows:

$$
O b j=O b j_{1}+O b j_{2}+O b j_{3} .
$$

As stated before, the TLBO is an algorithm used for maximizing an objective function, while for correct damage detection using objective function in Eq. (23), minimization is desired here. Therefore, the objective function applied in TLBO must be in the form of the maximizing problem as follows:

$$
O b j_{T L B O}=\frac{1}{1+O b j} .
$$

It is obvious that when $O b j_{T L B O}$ becomes maximum, the value of $O b j$ is minimum and damage parameters are determined.

\subsection{Numerical results}

In this section, the efficiency of the proposed objective function to detect the damage in a double-beam system is studied. As previously stated in the introduction, detecting damage is important in the early stages of local failure in the structures. Therefore, it is assumed that the number of damages in the doublebeam system could be one or two. It should be noted that there is not any limitation to the investigation of more damage in the proposed method. Three different kinds of damage occurrence have been studied that include: (a) the occurrence of damage while stiffness is decreasing in one of the elements, (b) the occurrence of a crack, and (c) the simultaneous occurrence of two damages. Further, the impact of some parameters on the accuracy of the proposed method is investigated. The population of the students in the TLBO is set to 25 through all examples. Whenever the value of the objective function becomes less than $10^{-10}$, the optimization algorithm is terminated and results of the algorithm are considered as damage parameters.

\subsubsection{Single damage as stiffness degradation}

Another case called stiffness degradation in one of the elements of beams or complete destruction in one of the elements of the inner elastic layer is considered here. In this case, the design variables of the optimization process include location and severity of damage. The whole system is divided into 36 elements; accordingly, elements 1 to 12 are related to the upper beam, elements 13 to 24 are related to the lower beam, and elements 25 to 36 are related to the core. Moreover, in the present algorithm, when the damaged element is detected in the core, the severity of the damage is considered as complete destruction of the element. The present method is successfully able to detect damage in different cases of location and severity of the damage. Now, this study examines the effect of the number of points of response measurement on the efficiency and convergence rate of the algorithm. The system response is measured one time at 10 points, as shown in Figure 4, and once more at 6 points, as shown in Figure 11. At first, the damage is considered in element 4 with a $6 \%$ decrease in the stiffness. The convergence of the objective function value is depicted in Figure 12. As shown, when the system response is measured at 10 points, the convergence rate will be faster in the algorithm. Another case considers damage in element 20 with a $10 \%$ reduction in the stiffness. The convergence of the objective function for this case is shown in Figure 13. Similar to the previous case, the convergence rate is faster when the response is measured at 10 points. In the case of the damage occurrence in the core, the convergence rate with 10 measurement points and 6 measurement points is almost the same. Although the convergence rate of the objective function is slow when the response is measured at 6 points, it should be considered that there

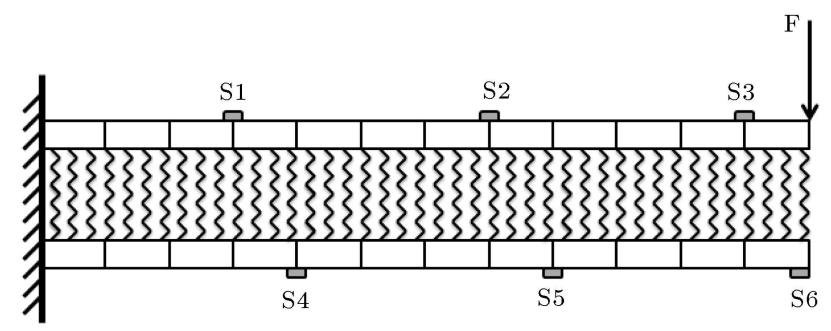

Figure 11. Configuration of the 6 measurement points in the double-beam system. 


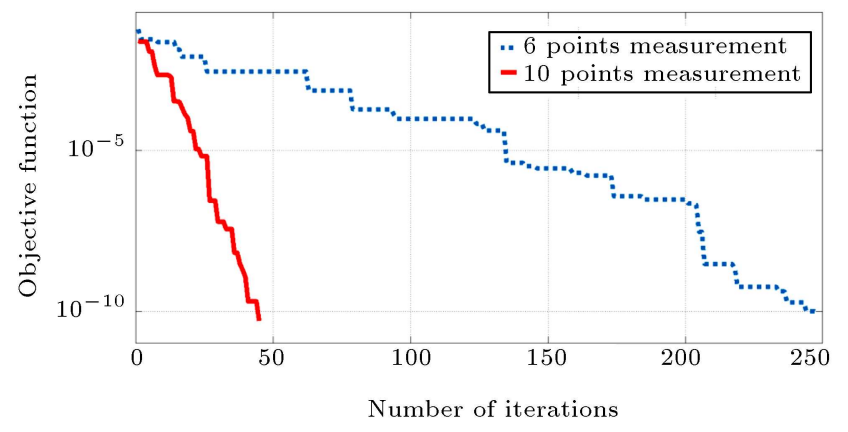

Figure 12. Convergence rate of the Teaching-Learning Based Optimization (TLBO) for different measurement points (damage is considered to be a $6 \%$ reduction in the stiffness of the element number 4).

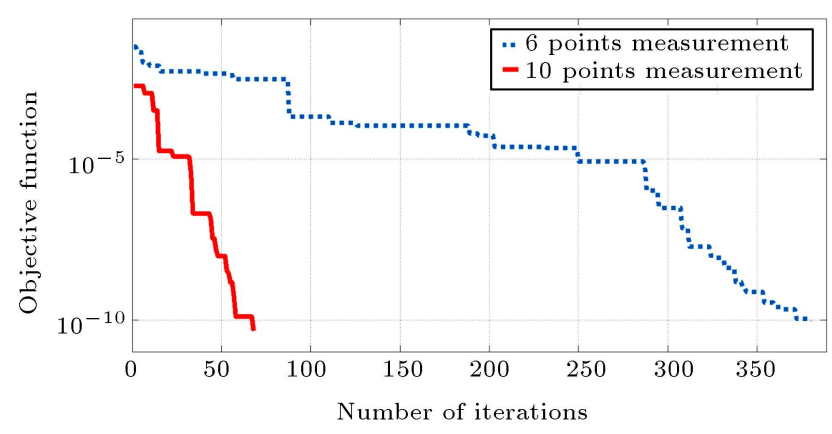

Figure 13. Convergence rate of the Teaching-Learning Based Optimization (TLBO) for different measurement points (damage is considered to be a $10 \%$ reduction in the stiffness of the element number 20).

is 48 degrees of freedom in the investigated structure (24 translational degrees and 24 rotational degrees) and the severity and location of single damage are detected with high accuracy only by response measurement at 6 points (less than $13 \%$ of the total degree of freedom), which could be considered as the advantage of the given objective function.

\subsubsection{Occurrence of the crack}

In this case, the design variables of the optimization process include the location and depth of the crack. The probable location of the crack varies from zero to 3 meters in depth. Based on crack location, the corresponding element in the finite element model can be easily calculated. The crack is placed from zero to 1.5 meters at the upper beam and from 1.5 to 3 meters at the lower beam; for example, when a crack occurs in the 1.8-meter location, its corresponding element is element 15. Eqs. (6) and (7) are used to extract the stiffness matrix of the cracked element. In this case, the response of the double-beam system is measured at 10 points, as presented in Figure 4. For different cases of the crack occurrence, the proposed algorithm is able to accurately identify the location and severity of the crack. Now, the effects of the excitation frequency

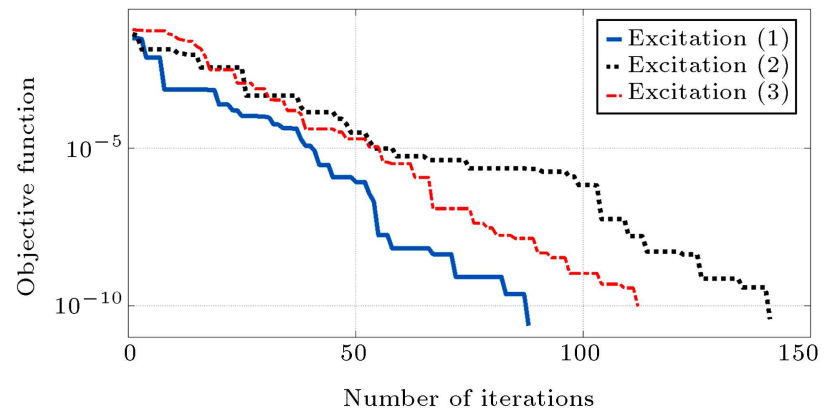

Figure 14. Convergence rate of the Teaching-Learning Based Optimization (TLBO) for different frequencies of excitation (crack at location $0.57 \mathrm{~m}$ with $\eta=0.08$ ).

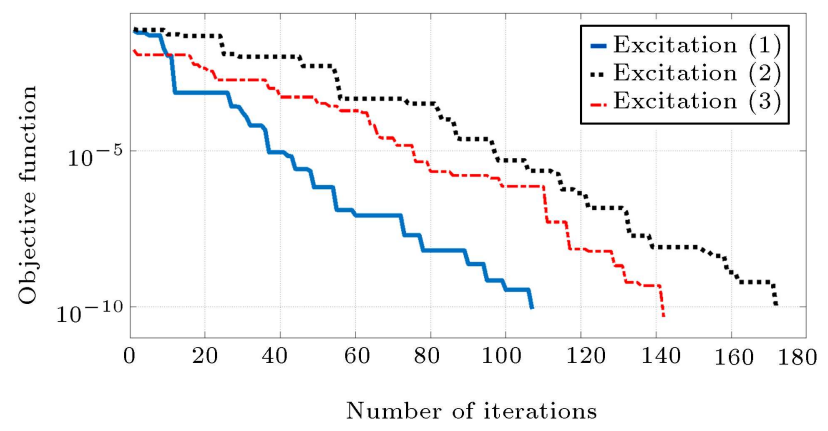

Figure 15. Convergence rate of the Teaching-Learning Based Optimization (TLBO) for different frequencies of excitation (crack at location $0.2 \mathrm{~m}$ with $\eta=0.05$ ).

on the accuracy and convergence rate of the proposed method are studied.

The first excitation frequency, Excitation (1), is lower than the first natural frequency of the system as in Eq. (18). The second excitation frequency, Excitation (2), is close to the first and second natural frequencies of the system and is considered as follows:

$$
F=2000 \sin (40 \pi t) .
$$

The third excitation frequency, Excitation (3), is close to the third and fourth frequencies of the system and is considered as follows:

$$
F=2000 \sin (240 \pi t)
$$

Four different cases are considered for the location and severity of the crack. The convergence rate of the algorithm for various excitation frequencies is presented in Figures 14 to 17. As is seen, the given algorithm is able to detect damage under different excitation frequencies. However, the effects of the excitation frequency on the convergence rate of the algorithm are sensible and definitely not ignorable.

\subsubsection{The simultaneous occurrence of two damages} In this case, the performance of the proposed objective function is investigated in the case of the simultaneous 


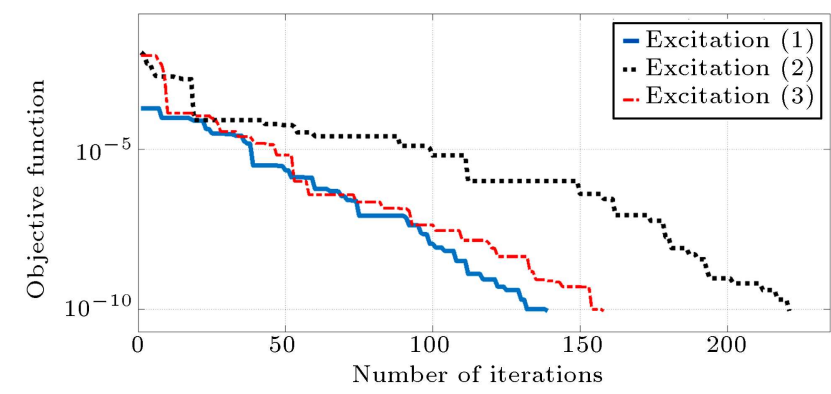

Figure 16. Convergence rate of the Teaching-Learning Based Optimization (TLBO) for different frequencies of excitation (crack at location $2.6 \mathrm{~m}$ with $\eta=0.1$ ).

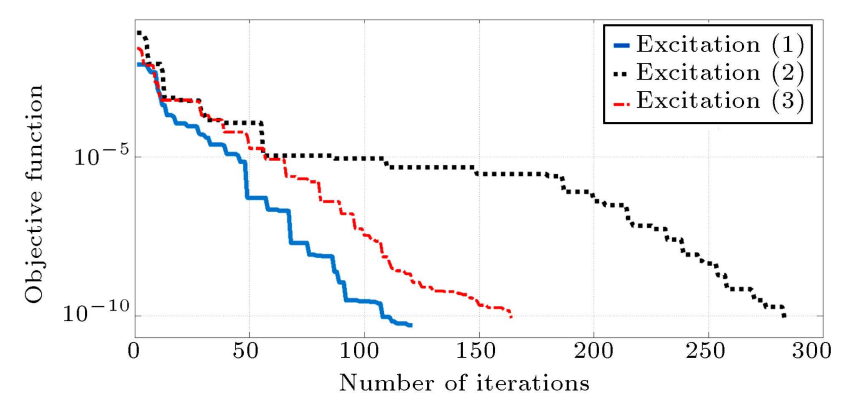

Figure 17. Convergence rate of the Teaching-Learning Based Optimization (TLBO) for different frequencies of excitation (crack at location $2.32 \mathrm{~m}$ with $\eta=0.15$ ).

detection of two damages. The number of measurement points of the response is 10 and similar to the arrangement shown in Figure 4. External excitation is considered in Eq. (18), and damage is shown to be

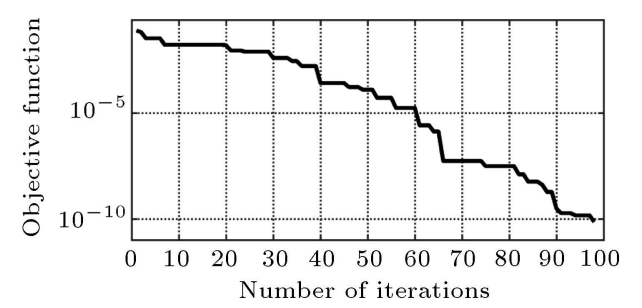

(a) reducing the stiffness of the beams' elements or the destruction of the core elements. In this case, the design variables of the optimization process are four, of which two variables are related to damage location and the other two correspond to the severity of damages. The efficiency of the algorithm for various cases is studied, and damages are identified with high accuracy. For four different cases of the damages, the convergence rate of the algorithm is investigated and depicted in Figures 18 and 19. It is clear that when one of the damages occurs in the core, the convergence rate of the algorithm becomes faster. Moreover, when the damage occurs at one of the elements of the lower beam near the free end, more iterations are required to obtain the correct parameters of damages.

\subsubsection{Noise immunity}

In this section, the effect of any error in measuring the acceleration or noise on the efficiency of the given method is discussed. Noise is added to the structural response in Eq. (21). The number of measurement points in the system's response is 10 and is similar to the arrangement of Figure 4. The external excitation is considered in Eq. (18). According to the obtained results of the previous sections, the optimization algorithm is terminated after 300 iterations, and its output considered is as damage. The noise applied to the measured response and the numerical model is random at each iteration of the algorithm. Damage represents the stiffness degradation of one of the beams' elements or core damage. The number of damage

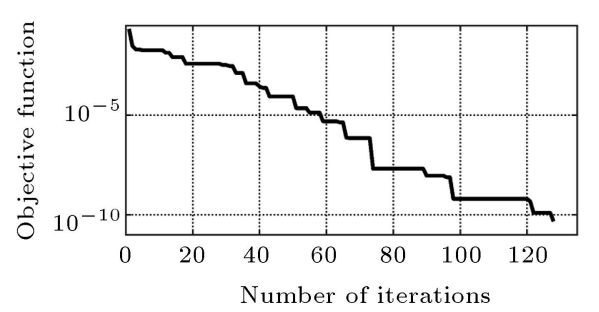

(b)

Figure 18. Convergence rate of the Teaching-Learning Based Optimization (TLBO) for the simultaneous occurrence of two damages: (a) 10\% stiffness reduction in the element number 6 and destruction of the element number 29 and (b) $10 \%$ stiffness reduction in the element number 19 and destruction of the element number 35.



(a)

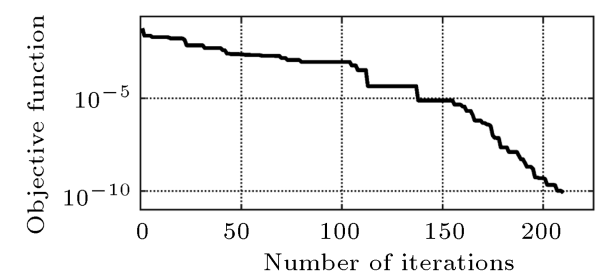

(b)

Figure 19. Convergence rate of the Teaching-Learning Based Optimization (TLBO) for the simultaneous occurrence of two damages: (a) $10 \%$ stiffness reduction in the element number 2 and a $15 \%$ stiffness reduction in the element number 8 and (b) $10 \%$ stiffness reduction in the element number 5 and a $10 \%$ stiffness reduction in the element number 20. 


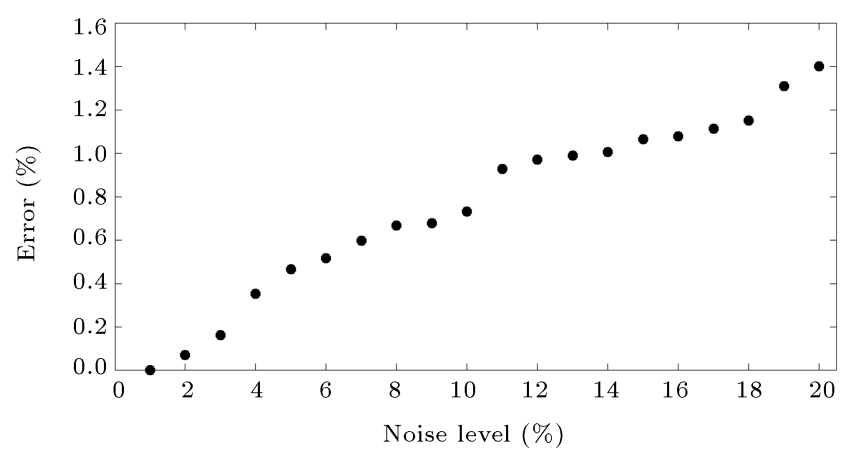

Figure 20. Error in identified severity of the damage versus the noise level (damage in element 3 with a $10 \%$ decrease in stiffness).

cases is assumed to be one or two. In the case of a single damage case, when the damage occurs only at the core, the algorithm is able to detect damage at each noise level. When damage occurs in one of the elements of the beam, the damage location is correctly detected at each noise level. For the severity of detected damage, results are presented in Figures 20 and 21. The algorithm is performed three times at each noise level, and the average absolute error in detecting the severity of the damage is considered to be erroneous. Figure 20 shows the occurrence of damage in element 3 with a $10 \%$ decrease in stiffness. As seen, the algorithm detects the severity of the damage in high accuracy, even at a $20 \%$ noise level. Figure 21 shows the occurrence of the damage in element 20 with a $10 \%$ decrease in stiffness. Unlike Figure 20, in this case, the accuracy of the method is acceptable for the noise level up to $10 \%$. Now, this study investigates the simultaneous occurrence of the damage in the system. Here, when both of the damages occur at the core, the noise effect is not visible on the accuracy of the



Figure 21. Error in identified severity of the damage versus the noise level (damage in element 20 with a $10 \%$ decrease in stiffness).

algorithm and damage elements are easily detected. In other cases concerning the occurrence of two damages, the algorithm is executed three times and the best results are considered as damage parameters. In the case of the occurrence of single damage in beams' element and another damage at the core, results of the damage detection are shown in Table 2. As seen, the results are acceptable up to $10 \%$ noise level. Table 3 shows the occurrence of both damages to the beams. As seen, the accuracy of the method up to $5 \%$ noise level is independent of damages' location. For the noise level higher than $5 \%$, damage detection results of damages near the clamp end are almost accurate up to $10 \%$ noise level or maybe more. However, when damages are located near the free end, by increasing the noise level, the reliability of the results decreases.

\section{Conclusion}

In the present study, the diagnosis of damage was investigated in a double-beam system. First, the numerical model of the system was formulated for various

Table 2. Noise effects on the accuracy of damage detection method (simultaneous occurrence of damages in beams and core).

\begin{tabular}{|c|c|c|c|c|c|c|c|c|c|c|}
\hline \multirow[b]{2}{*}{ Noise level } & & \multicolumn{5}{|c|}{ Scenario 1} & \multicolumn{4}{|c|}{ Scenario 2} \\
\hline & & Element no. & $\beta$ & Element & no. & $\bar{\beta}$ & Element no. & $\beta$ & Element no. & $\bar{\beta}$ \\
\hline- & Actual & 16 & 0.1 & 27 & & 1 & 9 & 0.1 & 33 & 1 \\
\hline $2 \%$ & Identified & 16 & 0.1002 & 27 & & 1 & 9 & 0.0988 & 33 & 1 \\
\hline $5 \%$ & Identified & 16 & 0.0993 & 27 & & 1 & 9 & 0.0975 & 33 & 1 \\
\hline $10 \%$ & Identified & 16 & 0.0968 & 27 & & 1 & 9 & 0.0951 & 33 & 1 \\
\hline
\end{tabular}

Table 3. Noise effects on the accuracy of damage detection method (multiple damages occurrence in beams).

\begin{tabular}{|c|c|c|c|c|c|c|c|c|c|}
\hline \multirow[b]{2}{*}{ Noise level } & & \multicolumn{4}{|c|}{ Scenario 1} & \multicolumn{4}{|c|}{ Scenario 2} \\
\hline & & Element no. & $\boldsymbol{\beta}$ & Element no. & $\beta$ & Element no. & $\boldsymbol{\beta}$ & Element no. & $\beta$ \\
\hline- & Actual & 5 & 0.1 & 15 & 0.1 & 17 & 0.1 & 21 & 0.1 \\
\hline $2 \%$ & Identified & 5 & 0.1004 & 15 & 0.1002 & 17 & 0.0995 & 21 & 0.1007 \\
\hline $5 \%$ & Identified & 5 & 0.1011 & 15 & 0.0997 & 17 & 0.0982 & 21 & 0.1054 \\
\hline $10 \%$ & Identified & 5 & 0.0972 & 15 & 0.1015 & 17 & 0.0954 & 21 & 0.1547 \\
\hline
\end{tabular}


damage types by applying the Finite Element Method (FEM). Then, the impact of damage occurrence at the upper beam, lower beam, and inner elastic layer on the Proper Orthogonal Value (POV) of the whole system was investigated. Since it was not possible to make a distinction between some damages such as damage to the upper beam near the clamped end and the damage to the inner layer near the free end through the POV of the entire system, the POV of each beam was calculated separately to obtain the ability of the various damage detections. Two objective functions were defined by using POV and Proper Orthogonal Mode (POM) of each beam separately. The noise applied to the system response was assumed to be a zero-mean vector; thus, an objective function was also defined by using the mean value of the time domain response of the system at all measurement points. Therefore, the value of this objective function does not change by changing the noise level. As a result of the three objective functions, a final objective function was obtained to detect damage in the system. The Teaching-Learning Based Optimization (TLBO) algorithm was used to solve the final objective function. In the noiseless mode, the algorithm successfully identified various types of damage that occurred at the beam or inner elastic layer, the occurrence of a crack, and the simultaneous occurrence of two damages. The effects of the number of points of system response measurement and the frequency of excitation on the efficiency and convergence rate of the algorithm were also investigated. The efficiency of the algorithm in damage detection was also investigated in the presence of noise, and acceptable results were obtained. The important points about the proposed method for damage detection in the double-beam system can be summarized as follows:

1. The proposed method only uses data from the time history response of the system and does not require frequency domain analysis;

2. By measuring the response of the system with 48 degrees of freedom at 10 points (less than $21 \%$ of the total degrees of freedom), various types of damage occurrence including one or two damage cases were detected with acceptable accuracy, even in the presence of noise;

3. The precision of this method is independent of the frequency of the excitation, although the frequency of excitation is conducive to the convergence rate of the algorithm;

4. According to the results of Ref. [19], a partial removal of the elastic layer was considered as damage at the core did not affect the odd mode frequencies of the system; consequently, through the usual methods of modal parameters, detecting the core damage is not easily possible and, so far, no research has been done in this area, while damage at the core is detected by the current method, even in the presence of excessive noise;

5. The accuracy of the method in the presence of noise depends on the location of the damage. In some cases, this method was able to detect damage with high accuracy, even up to $20 \%$ noise level.

\section{References}

1. Fan, W. and Qiao, P. "Vibration-based damage identification methods: a review and comparative study", Structural Health Monitoring, 10, pp. 83-111 (2011).

2. Ruotolo, R. and Surace, C. "Damage assessment of multiple cracked beams: numerical results and experimental validation", Journal of Sound and Vibration, 206(4), pp. 567-588 (1997).

3. Meruane, V. and Heylen, W. "An hybrid real genetic algorithm to detect structural damage using modal properties", Mechanical Systems and Signal Processing, 25(5), pp. 1559-1573 (2011).

4. Raich, A.M. and Liszkai, T.R. "Improving the performance of structural damage detection methods using advanced genetic algorithms", Journal of Structural Engineering, 133(3), pp. 449-461 (2007).

5. Dabbagh, H., Ghodrati Amiri, G., and Shaabani, S. "Modal data-based approach to structural damage identification by means of imperialist competitive optimization algorithm", Scientia Iranica, 25(3), pp. 1070-1082 (2018).

6. Kaveh, A., Hosseini Vaez, S.R., and Hosseini, P. "Enhanced vibrating particles system algorithm for damage identification of truss structures", Scientia Iranica, 20(1), pp. 246-256 (2019). DOI: $10.24200 /$ sci. 2017.4265

7. Seyedpoor, S.M., Shahbandeh, S., and Yazdanpanah, O. "An efficient method for structural damage detection using a differential evolution algorithm-based optimisation approach", Civil Engineering and Environmental Systems, 32(3), pp. 230-250 (2015).

8. Fatahi, L. and Moradi, S. "Multiple crack identification in frame structures using a hybrid Bayesian model class selection and swarm-based optimization methods", Structural Health Monitoring, 17, pp. 39-58 (2018).

9. Fallahian, S., Joghataie, A., and Kazemi, M.T. "Structural damage detection using time domain responses and teaching-learning-based optimization (TLBO) algorithm", Scientia Iranica, 25(6), pp. 3088-3100 (2018). DOI: $10.24200 /$ sci.2017.4238

10. Rezvani, K., Maia, N.M.M., and Sabour, M.H. "A comparison of some methods for structural damage detection", Scientia Iranica, 25(3), pp. 1312-1322 (2018). 
11. Cao, S. and Ouyang, H. "Robust multi-damage localisation using common eigenvector analysis and covariance matrix changes", Mechanical Systems and Signal Processing, 111, pp. 663-677 (2018).

12. Civera, M., Zanotti Fragonara, L., and Surace, C. "A novel approach to damage localisation based on bispectral analysis and neural network", Smart Structures and Systems, 20(6), pp. 669-682 (2017).

13. Oniszczuk, Z. "Free transverse vibrations of elastically connected simply supported double-beam complex system", Journal of Sound and Vibration, 232(2), pp. 387-403 (2000).

14. Palmeri, A. and Adhikari, S. "A Galerkin-type statespace approach for transverse vibrations of slender double-beam systems with viscoelastic inner layer", Journal of Sound and Vibration, 330, pp. 6372-6386 (2011).

15. Stojanovic, V., Kozic, P., and Janevski, G. "Exact closed-form solutions for the natural frequencies and stability of elastically connected multiple beam system using Timoshenko and high-order shear deformation theory", Journal of Sound and Vibration, 332, pp. 563-576 (2013).

16. Huang, M. and Liu, J.K. "Substructural method for vibration analysis of the elastically connected doublebeam system", Advances in Structural Engineering, 16(2), pp. 365-377 (2013).

17. Mirzabeigy, A., Dabbagh, V., and Madoliat, R. "Explicit formulation for natural frequencies of doublebeam system with arbitrary boundary conditions", Journal of Mechanical Science and Technology, 31(2), pp. 515-521 (2017).

18. Mirzabeigy, A., Madoliat, R., and Vahabi, M. "Free vibration analysis of two parallel beams connected together through variable stiffness elastic layer with elastically restrained ends", Advances in Structural Engineering, 20(3), pp. 275-287 (2017).

19. Mirzabeigy, A. and Madoliat, R. "Free vibration analysis of partially connected parallel beams with elastically restrained ends", Proceedings of the Institution of Mechanical Engineers, Part C: Journal of Mechanical Engineering Science, 230(16), pp. 2851-2864 (2016).

20. Nguyen, K.V. "Crack detection of a double-beam carrying a concentrated mass", Mechanics Research Communications, 75, pp. 20-28 (2016).

21. Liu, G.R. and Quek, S.S., The Finite Element Method: A Practical Course, Butterworth-Heinemann (2013).

22. Newmark, N.M. "A method of computation for structural dynamics", Journal of the Engineering Mechanics Division, 85(3), pp. 67-94 (1959).
23. Mehrjoo, M. Khaji, N., and Ghafory-Ashtiany, M. "Application of genetic algorithm in crack detection of beam-like structures using a new cracked EulerBernoulli beam element", Applied Soft Computing, 13(2), pp. 867-880 (2013).

24. Ostachowicz, W.M. and Krawczuk, M. "Analysis of the effect of cracks on the natural frequencies of a cantilever beam", Journal of Sound and Vibration, 150(2), pp. 191-201 (1991).

25. Rao, R.V., Savsani, V.J., and Vakharia, D.P. "Teaching-learning-based optimization: an optimization method for continuous non-linear large scale problems", Information Sciences, 183(1), pp. 1-15 (2012).

26. Singh, M., Panigrahi, B.K., and Abhyankar, A.R. "Optimal coordination of directional over-current relays using teaching learning-based optimization (TLBO) algorithm", International Journal of Electrical Power \& Energy Systems, 50, pp. 33-41 (2013).

27. García, J.A.M. and Mena, A.J.G. "Optimal distributed generation location and size using a modified teaching-learning based optimization algorithm", International Journal of Electrical Power \& Energy Systems, 50, pp. 65-75 (2013).

28. Basu, M. "Teaching-learning-based optimization algorithm for multi-area economic dispatch", Energy, 68, pp. 21-28 (2014).

29. Jordehi, A.R. "Optimal setting of TCSCs in power systems using teaching-learning-based optimisation algorithm", Neural Computing and Applications, 26(5), pp. 1249-1256 (2015).

30. Farshchin, M., Camp, C.V., and Maniat, M. "Multiclass teaching-learning-based optimization for truss design with frequency constraints", Engineering Structures, 106, pp. 355-369 (2016).

31. Kerschen, G., Golinval, J.C., Vakakis, A.F., and Bergman, L.A. "The method of proper orthogonal decomposition for dynamical characterization and order reduction of mechanical systems: an overview", Nonlinear Dynamics, 41(1), pp. 147-169 (2005).

32. Kerschen, G., Poncelet, F., and Golinval, J.C. "Physical interpretation of independent component analysis in structural dynamics", Mechanical Systems and Signal Processing, 21(4), pp. 1561-1575 (2007).

33. Feeny, B.F. and Kappagantu, R. "On the physical interpretation of proper orthogonal modes in vibrations", Journal of Sound and Vibration, 211(4), pp. 607-616 (1998).

34. Kerschen, G. and Golinval, J.C. "Physical interpretation of the proper orthogonal modes using the singular value decomposition", Journal of Sound and Vibration, 249(5), pp. 849-865 (2002).

35. Feeny, B.F. and Liang, Y. "Interpreting proper orthogonal modes of randomly excited vibration systems", Journal of Sound and Vibration, 265(5), pp. 953-966 (2003). 
36. Galvanetto, U. and Violaris, G. "Numerical investigation of a new damage detection method based on proper orthogonal decomposition", Mechanical Systems and Signal Processing, 21(3), pp. 1346-1361 (2007).

37. Galvanetto, U., Surace, C., and Tassotti, A. "Structural damage detection based on proper orthogonal decomposition: experimental verification", AIAA Journal, 46(7), pp. 1624-1630 (2008).

38. Thiene, M., Zaccariotto, M., and Galvanetto, U. "Application of proper orthogonal decomposition to damage detection in homogeneous plates and composite beams", Journal of Engineering Mechanics, 139(11), pp. 1539-1550 (2013).

39. Rao, A.R.M., Lakshmi, K., and Venkatachalam, D. "Damage diagnostic technique for structural health monitoring using POD and self adaptive differential evolution algorithm", Computers \& Structures, 106, pp. 228-244 (2012).

\section{Biographies}

Alborz Mirzabeigy received his BS degree in Mechanical Engineering from Iran University of Science and Technology, Tehran, Iran and his MS degree in Mechanical Engineering from Amirkabir University of Technology (Tehran Polytechnic), Tehran, Iran. He is currently a PhD Candidate at Iran University of Science and Technology, Tehran, Iran. His research interests include vibration-based techniques in damage detection, computational methods, applied mathematics, passive control of structures, and inverse problems.

Reza Madoliat received his $\mathrm{MS}$ and $\mathrm{PhD}$ degrees from Michigan State University, USA. His main research interests include inverse problems, machinery vibration, and numerical analysis. He is currently an Associate Professor in the School of Mechanical Engineering, Iran University of Science and Technology, Tehran, Iran. 